\title{
Impedance Measurement of Batteries under load
}

\author{
Rahat Hasan \\ School of Engineering \\ University of Waikato, Hamilton, New Zealand
}

\author{
Professor Jonathan Scott \\ School of Engineering \\ University of Waikato, Hamilton, New Zealand
}

\begin{abstract}
The impedance of a lithium battery at a single state-of-charge $(\mathrm{SoC})$ is obtained from the current and voltage response of the cell. Making this measurement is important in order to understand cell characteristics. There exist some impedance analysers which can make suitable measurements at a single SoC. Measuring this impedance at multiple SoC is slow and tedious especially at lower frequencies. We present a new method which computes the impedance over a range of SoC while simultaneously varying the SoC. The proposed method produces reliable data even when the device under test is behaving nonlinearly. The data obtained from this measurement is validated by comparing impedance data obtained at different $\mathrm{SoC}$ from a cell under no load.
\end{abstract}

Index Terms-batteries, impedance measurement, frequency domain analysis, state-of-charge

\section{INTRODUCTION}

In recent years, there is an increasing interest in measuring the impedance of batteries over a range of frequencies [1][4]. Most commonly the impedance is measured using the I$\mathrm{V}$ method which observes the current and response voltage signals. The impedance is then obtained by dividing the complex voltage by the complex current.

There exist instruments which make this measurement [5], [6]. A more robust method of measuring impedance was proposed which yields accurate data at extremely low frequencies when measuring non-linear components via adjusting the stimuli level at each frequency [7]. All of these methods assume that the battery is disconnected from the load.

Most authors propose battery models [8]-[14] mainly for the purpose of estimating the state-of-charge and state-of-health. All these models were produced from frequency response data obtained at a single SoC. A typical impedance plot of a single $800 \mathrm{mAh} \mathrm{Li-ion} \mathrm{cell} \mathrm{is} \mathrm{shown} \mathrm{in} \mathrm{figure} 1$. The steps used to produce figure 1 is adopted from paper [7]. It is seen from this simple curve that a battery resembles a pure resistor at higher frequencies but depicts the behaviour of a capacitor (possibly fractional) at lower frequencies.

The impedance of a battery is subject to change depending on external factors such as temparature and SoC. Paper [15] inspects how the impedance of a $\mathrm{LiFePo} 4$ battery varies with temperature at different state of charge. On the other hand, authors of paper [16] speculate how the impedance changes with the SoC. They estimated this parametric change from a fractional-order derivatives using recursive least square technique.

In this paper, we propose a method which can measure the impedance of a cell under load while varying the SoC. Sec- tion II investigates the current method of measuring impedance at a single SoC. Section III gives a tutorial of an alternative method which measures impedance while the SoC of a cell is varied. Section IV proposes our conclusion.

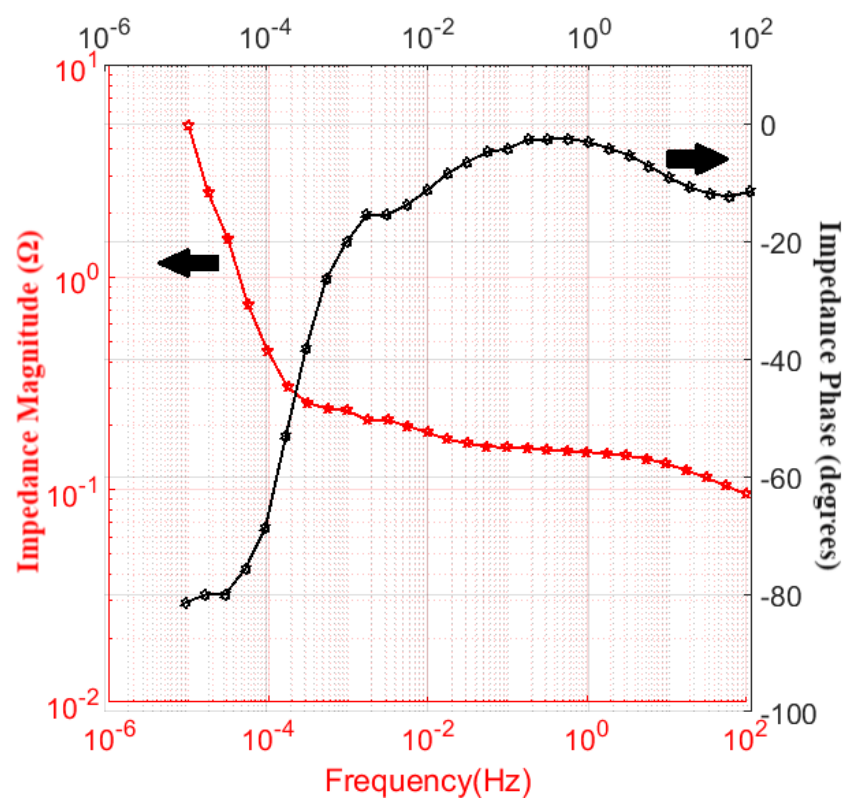

Fig. 1. Impedance curve of a $800 \mathrm{mAh} \mathrm{Li-ion} \mathrm{cell.}$

\section{TRADITIONAL METHOD}

Traditionally, the impedance of a cell is measured under no load at a single SoC. To measure impedance at different SoC, first a certain amount of charge is drawn out of the cell over a period of time. A small current stimuli is then applied and the response voltage across the cell is measured under no load. The impedance of the cell is then calculated and stored. This procedure is continued until the cell is fully discharged.

We reproduced this method on a $800 \mathrm{mAh} \mathrm{Li-ion} \mathrm{cell.} \mathrm{The}$ external temperature of the battery is maintained at 25 degrees Celsius throughout the measurement using a Contherm Polar 1000. We used an Agilent E5270 with E5281A source/monitor to make this measurement. The source monitor unit (SMU) was programmed in python. The connection is shown in figure 2. The steps followed are as below:

1) Use a current source to charge the cell to its full capacity.

2) Rest for 30 minutes to ensure that the chemistry inside the cell is stable. 


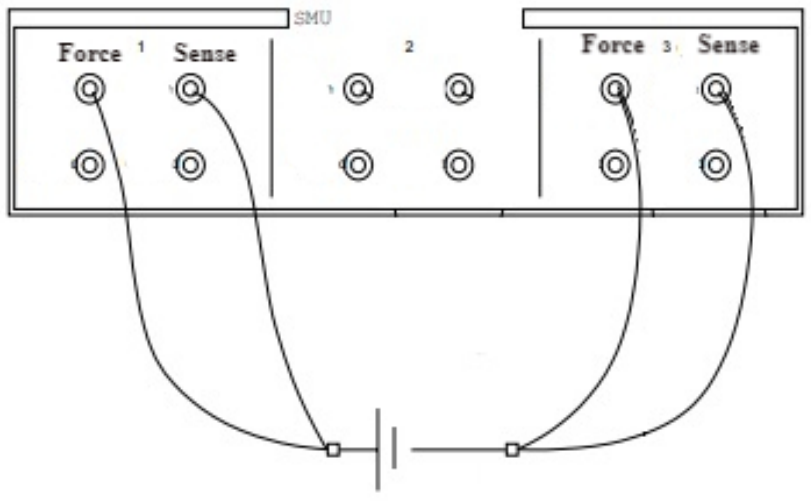

Fig. 2. The setup used to measure impedance of Li-ion cell at different SoC.

3) Draw $0.1 \mathrm{~A}$ for 120 seconds. This is equivalent to drawing 12 Coulombs or $0.4 \%$ of charge.

4) Rest for 30 seconds.

5) Reproduce the impedance measurement steps at $10 \mathrm{mHz}$ and $1 \mathrm{mHz}$ from paper [7].

6) Store the measured impedance in a data file.

7) Repeat steps 3-6 until the cell is fully discharged.

Figure 3 presents the result. We speculate the quantization of the phase data at $10 \mathrm{mHz}$ is a property of the cell. Although we know that impedance phase data is more prone to noise than impedance magnitude data.

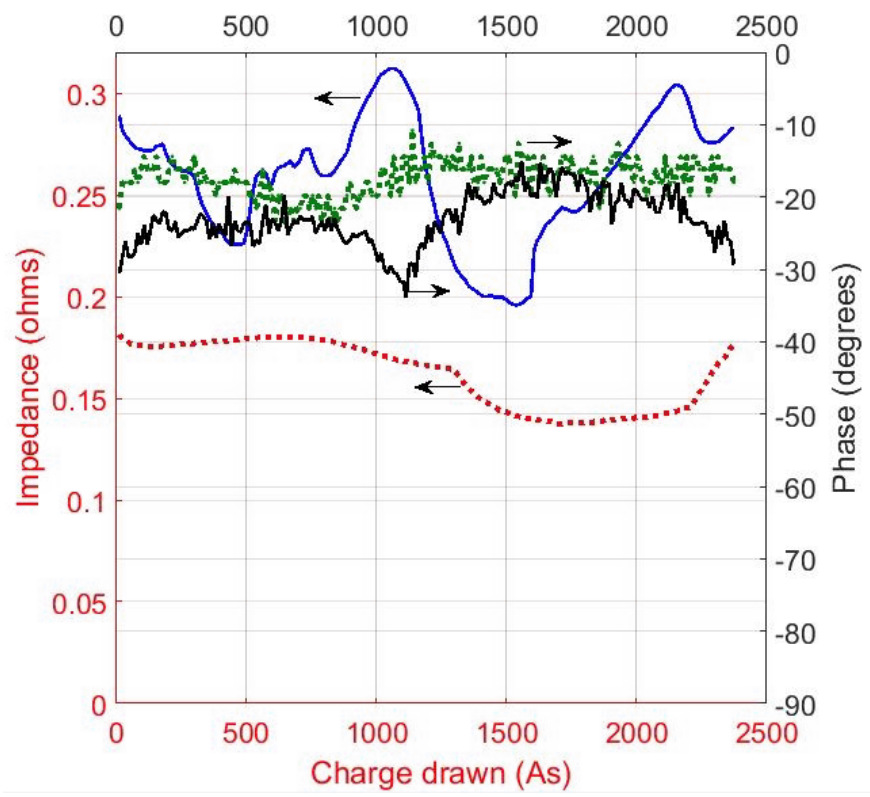

Fig. 3. Impedance magnitude and phase measured using traditional method under no load at different $\mathrm{SoC}$ for a Li-ion cell at $10 \mathrm{mHz}$ (dotted lines) and $1 \mathrm{mHz}$ (solid lines).

\section{PROPOSED METHOD}

In this section, we discuss a method which measures the impedance of a battery while simultaneously varying the
SoC. We used a programmable current source to produce a sinusoidal current with a DC offset. Such measurement can be performed by a range of power supplies which can sink and source current such as Hameg HM8143 twoquadrant power supplies, Tektronix (Keithley) 2400-series Source-Mearurement Units (SMUs), Keysight Precision I/V Analyzers, and Chroma 17000-series Programmable Battery Charge/Discharge Test Systems. We will show that this alternative method is superior because it conducts an impedance measurement at frequencies below $1 \mathrm{mHz}$ while simultaneously altering the SoC.

\section{A. Selecting the parameters of the current stimuli}

To measure the impedance of a cell over a range of SoC, a small sine-varying current signal with a DC offset is applied. The response voltage across the cell is measured simultaneously. The time domain equation of the current signal is

$$
I=I_{0} \sin (2 \pi f t)-I_{o s}
$$

Where $I_{0}$ is the amplitude of the signal, $f$ is the desired frequency and $I_{O S}$ is the negative offset of the signal. The integral of the positive and negative half of the cycle will yield the amount of charge transferred to and from the cell respectively. For the purpose of the calculation, we denote the charge transferred to the cell as $Q_{i n}$ and charge moved out the cell as $Q_{\text {out }}$.

$$
Q_{i n}=\int_{0}^{T / 2} I d t=\int_{0}^{T / 2} I_{0} \sin (2 \pi f t)-I_{o s} d t
$$

and

$$
Q_{\text {out }}=\int_{T / 2}^{T} I d t=\int_{T / 2}^{T} I_{0} \sin (2 \pi f t)-I_{\text {os }} d t
$$

Integration of equations 2 and 3 gives

$$
Q_{i n}=-\frac{I_{0}}{2 \pi f}[\cos (\pi f T)-1]-\frac{I_{o s} T}{2}
$$

and similarly

$$
Q_{\text {out }}=-\frac{I_{0}}{2 \pi f}[\cos (2 \pi f T)-\cos (\pi f T)]-\frac{I_{o s} T}{2}
$$

substituting $f T=1$ leads to

$$
Q_{i n}=\frac{I_{0}}{\pi f}-\frac{I_{o s}}{2 f}
$$

and

$$
Q_{\text {out }}=-\frac{I_{0}}{\pi f}-\frac{I_{\text {os }}}{2 f}
$$

In both equations 6 and 7 , the term $\frac{I_{o s}}{2 f}$ determines the discharging rate of the cell and $\frac{I_{0}}{\pi f}$ decides the nature of the sinusoidal signal. Since both terms are dependant on the frequency of the signal, the offset and amplitude of the current signal are selected accordingly for each frequency. In all our measurement, we limited the charge flow in the cell at \pm 10 $\%$ of the total cell charge. 


\section{B. Proposed method}

The steps for the measurement procedures are listed as follows:

1) Use a current source to charge the cell to its full capacity.

2) Rest for 30 minutes to ensure that the chemistry inside the cell is stable.

3) Use current drive to generate the stimulus signal.

4) Fix the amplitude and the offset of the signal at desired frequency according to subsection III-A.

5) Execute the measurement over at least 100 cycles until the cell is fully discharged. Using equations 6 and 7, select appropriate current stimuli level at each frequency to maintain the $\mathrm{SoC}$ within $10 \%$ in either direction.

6) Slice the data into $n$ number of cycles and save the input current and measured response voltage data in a text file for further analysis.

7) Apply hann window to each data slice to compensate for truncation.

8) Obtain the complex magnitude and phase of the current and voltage signal using Fourier Transform at the signal frequency.

9) Divide the complex voltage by the complex current to calculate the impedance of the cell.

C. Measuring impedance over a range of SoC of a single Liion battery

We next measured the impedance of a $800 \mathrm{mAh} \mathrm{Li-ion}$ cell over a range of SoC using our proposed method. We programmed the SMUs of Agilent E5270A with python to produce the stimuli at $10 \mathrm{mHz}$ and $1 \mathrm{mHz}$. The connection used in our proposed method is still the same as the traditional one. Appropriate amplitude and offset of the stimuli were chosen which will cycle the cell 200 times before it gets fully discharged. Throughout the entire measurement, the external temperature of the battery is kept constant as before. Figure 4 depicts the first 30 cycles of the current and response voltage waveform measured at $10 \mathrm{mHz}$. Readers may notice the need for windowing the response voltage data prior Fourier Transform since the end points of a cycle are not the same.

Figure 5 shows the new impedance magnitude and phase measured at different SoC using our method. The magnitude and the phase change significantly at different $\mathrm{SoC}$ as the frequency gets smaller. This is expected since batteries only exhibit interesting behaviour at extremely low frequencies. At $1 \mathrm{mHz}$, the percentage difference between the impedance obtained using our method and the traditional method seen previously in figure 2 is within $10 \%$. We attributed the discrepancies in the noisy phase data obtained using traditional method to instrument noise and uncertainties.

\section{CONCLUSION}

In this paper, we have shown there are other ways to measure the impedance of a battery over a range of SoC. Our method is superior to the existing one because it can measure impedance for a given cell while smoothly varying the SoC. Unlike the traditional method, this new technique

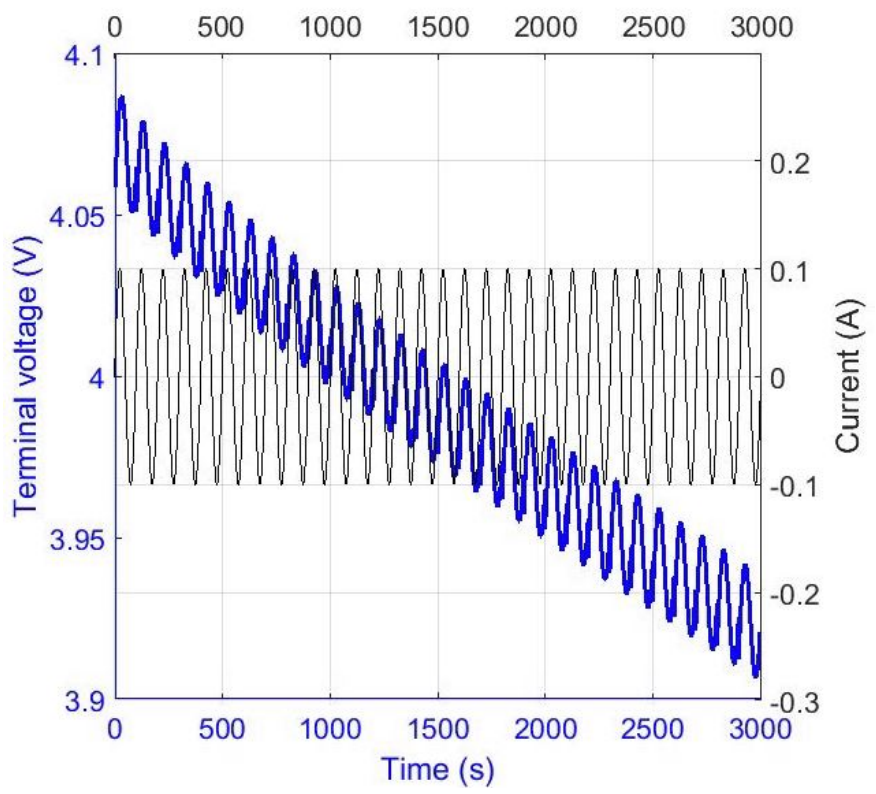

Fig. 4. Time-domain current and response voltage values obtained at $10 \mathrm{mHz}$ stimulus frequency.

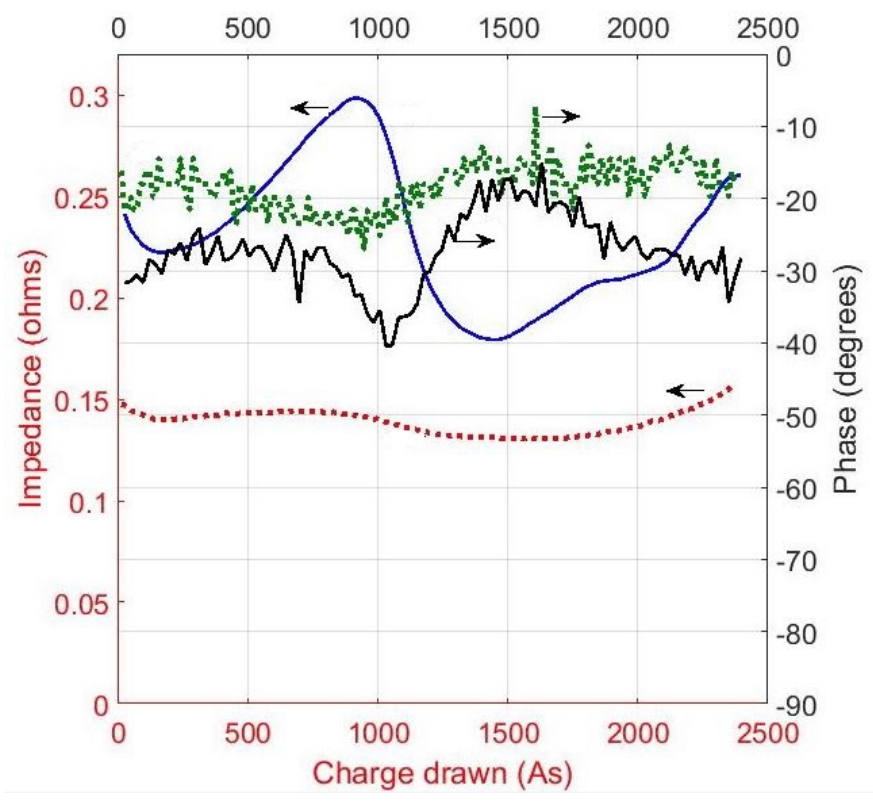

Fig. 5. Impedance magnitude and phase measured under load in comparison to figure 3 using our proposed method at different SoC for a Li-ion cell at $10 \mathrm{mHz}$ (dotted lines) and $1 \mathrm{mHz}$ (solid lines).

measures the impedance of a cell under load. Data was shown to be valid by comparison with data obtained using the traditional method. The percentage difference between the impedance obtained using the two method is within $10 \%$ at $1 \mathrm{mHz}$. The only drawback of this method is its limitation of measuring impedance at extremely low frequencies where noise is introduced in the measured data due to low stimuli level. 


\section{REFERENCES}

[1] Jaber A. Abu Qahouq, "Online battery impedance spectrum measurement method", 2016 IEEE Applied Power Electronics Conference and Exposition (APEC), 2016, pages: 3611 - 3615.

[2] Md. Kamal Hossain, S.M. Rakiul Islam, "Battery Impedance Measurement Using Electrochemical Impedance Spectroscopy Board", 2017 2nd International Conference on Electrical and Electronic Engineering (ICEEE), 2017, pages: 1 - 4.

[3] David A. Howey, Paul D. Mitcheson, Vladimir Yufit, Gregory J. Offer, Nigel P. Brandon, "Online Measurement of Battery Impedance Using Motor Controller Excitation", IEEE Transactions on Vehicular Technology, 2014, Volume: 63 , Issue: 6, pages: 2557 - 2566.

[4] Yuan Cao, Jaber A. Abu Qahouq, "Evaluation of paralleled battery system with SOC balancing and battery impedance magnitude measurement", 2018 IEEE Applied Power Electronics Conference and Exposition (APEC), 2018, pages: 437 - 441.

[5] Solartron 1260A Impedance/Gain-Phase Analyzer, Ametek Scientific Instruments. Accessed July, 2018. [Online]. Available: https://www.ameteksi.com/products/frequency-response-analyzers/ 1260a-impedance-analyzer.

[6] MFIA User Manual, Zurich Instruments AG. Accessed October, 2018. [Online]. Available: https://www.zhinst.com/sites/default/files/ziMFIA_UserManual_53400. pdf.

[7] Hasan, R., and Scott, J. B., "Measurement of Battery Impedance Extending to Extremely Low Frequencies", IEEE Transactions on Industrial Electronics, submitted.

[8] Jocelyn Sabatier, Mohamed Aoun, Alain Oustaloup, Gilles Gregoire, Frank Ragot and Patrick Roy, "Fractional system identification for lead acid battery state of charge estimation", Signal Processing Fractional calculus applications in signals and systems, Vol. 86, Issue 10, October 2006, pp.2645-2657.

[9] Yan Ma, Xiuwen Zhou, and Hong Chen,"Fractional Modeling and SOC Estimation of Lithium-ion Battery", IEEE/CAA Journal of Automatica Sinica, Vol 3, No. 3, July 2016, pp. 281-287.

[10] Jocelyn Sabatier, Mathieu Merveillaut, Junior Mbala Francisco, Franck Guillemard, Denis Porcelatto, "Fractional models for lithium-ion batteries”, 2013 European Control Conference (ECC), Zrich, Switzerland, July 2013, pp. 3458-3463.

[11] Luiz Carlos Stevanatto, Valner Joo Brusamarello, and Stanislav Tairov, "Parameter Identication and Analysis of Uncertainties in Measurements of Lead Acid Batteries", IEEE TRANSACTIONS ON INSTRUMENTATION AND MEASUREMENT, VOL. 63, NO. 4, APRIL 2014, pp. 761768.

[12] J. Chiasson and B. Vairamohan, "Estimating the state of charge of a battery", IEEE Transactions on Control Systems Technology, volume 13, Issue 3, May 2005, pp: 465-470.

[13] Tiezhou Wu, Lunan Liu, Qing Xiao, Quan Cao, and Xieyang Wang, "Research on SoC Estimation Based on Second-order RC Model", Telkomnika, vol. 10, no. 7, Nov 2012, pp. 1667-1672.

[14] ZeCheng, JikaoLv, YanliLiu, and ZhihaoYan, "Estimation of State of Charge for Lithium-Ion Battery Based on Finite Difference Extended Kalman Filter, Journal of Applied Mathematics, 2014, Vol 2014, pp. $1-10$.

[15] Luc H. J. Raijmakers, Dmitri L. Danilov, Joop P. M. van Lammeren, Thieu J. G. Lammers, Henk Jan Bergveld, Peter H. L. Notten, "Non-Zero Intercept Frequency: An Accurate Method to Determine the Integral Temperature of Li-Ion Batteries", IEEE Transactions on Industrial Electronics, 2016, Volume: 63, Issue: 5, pages: 3168 - 3178.

[16] Arijit Guha, and Amit Patra, "Online Estimation of the Electrochemical Impedance Spectrum and Remaining Useful Life of LithiumIon Batteries", IEEE TRANSACTIONS ON INSTRUMENTATION AND MEASUREMENT, VOL. 67, NO. 8, AUGUST 2018, pp. 1836-1849. 